\title{
Predicted term energies, wavelengths and oscillator strengths for transitions involving high- $n l$ Rydberg states in C II, C III and C IV
}

\author{
P. Quinet ${ }^{\star}$ \\ Astrophysique et Spectroscopie, Université de Mons-Hainaut, B-7000 Mons, Belgium \\ Received October 14; accepted November 10, 1997
}

\begin{abstract}
Predicted term energies for values of the principal quantum number up to 30 and orbital quantum numbers $l \geq 3$ in C II, C III and C IV have been obtained using the Ritz and the polarization formulae. Wavelength predictions for lines involving these high- $n l$ term energies together with the corresponding oscillator strengths, calculated by the HFR approach, are also reported in the present paper. Some astrophysical implications of these new atomic data are discussed by means of a comparison with the observed spectra of the planetary nebula NGC $7027^{1}$.
\end{abstract}

Key words: atomic data - planetary nebulae: NGC 7027

\section{Introduction}

Atomic dipole transitions involving high- $n l$ Rydberg states are frequently encountered in astrophysics and in laser physics. In astrophysics, the interpretation of recombination spectra emitted by extraterrestrial sources requires the knowledge of a large number of transition energies and rates as emphasized for example by HoangBinh et al. (1987) and Hoang-Binh (1990, 1993). In laser physics, oscillator strengths for transitions with large values of the principal quantum number $n$ can now be measured in laboratory by accurate techniques (see e.g. Connerade et al. 1992; Mende \& Kock 1996).

Recently, several dozens of lines involving intermediate and relatively large $(n, l)$ levels in the low ionization stages of very common elements such as carbon, nitrogen and oxygen were observed in the spectrum of the planetary nebula NGC 7027 between 399 and 1050 nm (Péquignot

\footnotetext{
* Collaborateur Scientifique F.N.R.S.

1 Tables 1 to 5 are also available, Tables 6 to 8 are only available in electronic form at the CDS via anonymous ftp to cdsarc.u-strasbg.fr (130.79.128.5).
}

\& Baluteau 1994; Baluteau et al. 1995; Péquignot 1996, 1997). More particularly, owing to the large abundance of carbon in NGC 7027, several Rydberg series of C II, C III and C IV were probably detected up to principal quantum number $n=30$ by Baluteau et al. (1995) and Péquignot $(1996,1997)$. In these papers, it is stated that available compilations of high- $n l$ atomic energy levels may not be sufficiently comprehensive to fully exploit the nebular spectroscopic data now accessible to common astrophysical devices and this lack of basic atomic data is pointed out as a major limitation to future progress in the field of nebulae.

Up to now, the highest $(l \geq 3)$ Rydberg terms experimentally determined were $1 \mathrm{~s}^{2} 2 \mathrm{~s}^{2} 7 \mathrm{f}^{2} \mathrm{~F}^{\mathrm{o}}, 8 \mathrm{~g}{ }^{2} \mathrm{G}, 7 \mathrm{~h}{ }^{2} \mathrm{H}^{\mathrm{o}}$ in C II, $1 \mathrm{~s}^{2} 2 \mathrm{~s} 7 \mathrm{f}^{3} \mathrm{~F}^{\mathrm{o}}, 7 \mathrm{~g}{ }^{3} \mathrm{G}, 6 \mathrm{~h}{ }^{3} \mathrm{H}^{\mathrm{o}}$ in C III (Bashkin \& Stoner 1975) and $1 \mathrm{~s}^{2} 8 \mathrm{f}^{2} \mathrm{~F}^{\mathrm{o}}, 8 \mathrm{~g}{ }^{2} \mathrm{G}, 9 \mathrm{~h}{ }^{2} \mathrm{H}^{\mathrm{o}}, 9 \mathrm{i}{ }^{2} \mathrm{I}$ in $\mathrm{C}$ IV (Bashkin \& Stoner 1975; Tunklev et al. 1997). The aim of the present work is to provide predicted term energies for values of the principal quantum number up to 30 and orbital quantum numbers $l \geq 3$ in these three ions using the Ritz and the polarization formulae. At the same time, wavelength predictions to an accuracy of at least $0.01 \mathrm{~nm}$ for lines involving high- $n l$ Rydberg states together with the corresponding oscillator strengths, calculated with the help of the relativistic Hartree-Fock (HFR) method, are reported in the region $400-1100 \mathrm{~nm}$ which might be useful for the interpretation of astronomical observations.

\section{High- $n l$ term energies}

\subsection{The Ritz formula}

In $1 \mathrm{~s}^{2} 2 \mathrm{~s}^{2} n l$ (C II), $1 \mathrm{~s}^{2} 2 \mathrm{~s} n l$ (C III) and $1 \mathrm{~s}^{2} n l$ (C IV) configurations, the outer $n l$ electron moves in the resultant field of the nucleus and the inner electrons. In such cases, the deviations between the observed term energies and the exact hydrogen-like values, caused by small core-penetration and polarization effects, are accurately described by the Ritz formalism. In this semi-empirical 
technique, discussed in detail by Edlén (1964) and more recently by Drake (1994), the term energies are written as

$E(n, l)=E_{\mathrm{I}}-\frac{R y Z_{\mathrm{c}}{ }^{2}}{\left(n^{*}\right)^{2}}$

where $E_{\mathrm{I}}$ is the ionization energy, $R y$ is the reduced mass Rydberg constant $\left(R y=109732.3 \mathrm{~cm}^{-1}\right.$ for carbon), $Z_{\mathrm{c}}$ is the net charge of the core $\left(Z_{\mathrm{c}}=2,3,4\right.$ for C II, C III and $\mathrm{C}$ IV respectively) and $n^{*}$ is the effective principal quantum number given by

$n^{*}=n-\delta(n, l)$

$\delta(n, l)$ is the so-called quantum defect which is strongly dependent on the $l$-quantum number but only weakly on $n$ and which can be expressed, in the Ritz formalism, by

$\delta(n, l)=a+b\left(n^{*}\right)^{-2}+c\left(n^{*}\right)^{-4}+\ldots$

\subsection{The polarization formula}

The energies of high $l$ (non-penetrating) states can also be given to a good approximation by the polarization formula (Edlén 1964):

$E(n, l)=E_{\mathrm{I}}-E_{\mathrm{H}}-E_{\mathrm{R}}-E_{\mathrm{P}}$.

The hydrogenic, $E_{\mathrm{H}}$, and relativistic, $E_{\mathrm{R}}$, terms are given by

$$
\begin{aligned}
& E_{\mathrm{H}}=\frac{R y Z_{\mathrm{c}}^{2}}{n^{2}} \\
& E_{\mathrm{R}}=\frac{\alpha^{2} R y Z_{\mathrm{c}}^{4}}{n^{4}}\left(\frac{n}{l+1 / 2}-\frac{3}{4}\right)
\end{aligned}
$$

where $\alpha$ is the fine structure constant. The polarization term, $E_{\mathrm{P}}$, can be written as

$E_{\mathrm{P}}=A P(n, l)[1+k q(n, l)]$

with

$P(n, l)=R y \frac{3 n^{2}-l(l+1)}{2 n^{5}(l-1 / 2) l(l+1 / 2)(l+1)(l+3 / 2)}$

and

$$
q(n, l)=R y \times
$$

$\frac{35 n^{4}-5 n^{2}[6 l(l+1)-5]+3(l-1) l(l+1)(l+2)}{8 n^{7}(l-3 / 2)(l-1)(l-1 / 2) l(l+1 / 2)(l+1)(l+3 / 2)(l+2)(l+5 / 2) P(n, l)}$.

In Eq. (7), the factors $A$ and $k$ can be expressed respectively by
$A=\alpha_{\mathrm{d}} Z_{\mathrm{c}}^{4}$

and

$k=\frac{\alpha_{\mathrm{q}}}{\alpha_{\mathrm{d}}} Z_{\mathrm{c}}^{2}$

where $\alpha_{\mathrm{d}}$ is the dipole polarizability of the atomic core in units of $a_{0}{ }^{3}$ and $\alpha_{\mathrm{q}}$ represents the quadrupole polarizability of the core in units of $a_{0}^{5}$.

\subsection{Predicted term energies}

Using the values 196664.7 and $386241.0 \mathrm{~cm}^{-1}$ (Moore 1970) for the ionization energies of C II and C III respectively and the observed term energies deduced from the compilation of Bashkin \& Stoner (1975), new Ritz formulae were derived for the $1 \mathrm{~s}^{2} 2 \mathrm{~s}^{2} n \mathrm{f}, 1 \mathrm{~s}^{2} 2 \mathrm{~s}^{2} n \mathrm{~g}$ (C II) and $1 \mathrm{~s}^{2} 2 \mathrm{sng}$ (C III) series. The Ritz parameters (a, b, c) obtained in the present work as well as the standard deviations between the experimental term energies and those calculated from the Ritz formulae are given in Table 1. For the $1 \mathrm{~s}^{2} 2 \mathrm{~s}^{2} n \mathrm{~h}-n \mathrm{i}$ (C II), $1 \mathrm{~s}^{2} 2 \mathrm{~s} n h-n \mathrm{k}$ (C III) and $1 \mathrm{~s}^{2} n \mathrm{f}-n \mathrm{k}$ (C IV) series, the polarization Eq. (4) was used. In the cases of C II and C III, only the dipole contribution was retained ( $k$ was set equal to zero) with the dipole polarizabilities $\alpha_{\mathrm{d}}=3.77$ and $3.46 a_{0}^{3}$ respectively. These values were derived from the oscillator strengths published for C III and C IV using the following formula:

$\alpha_{\mathrm{d}}=4 a_{0}^{3} \sum_{i} \frac{f_{0 i}}{\left(E_{i}-E_{0} / R y\right)^{2}}$

where $\left(E_{i}-E_{0} / R y\right)$ are the transition energies (in Rydbergs) for lines connecting the ground level in C III and $\mathrm{C}$ IV and $f_{0 i}$ the corresponding oscillator strengths taken from the Opacity Project (the Opacity Project Team 1995) and corrected with experimental wavelengths by Verner et al. (1994). In the case of C IV, we used the polarization Eq. (4) including both dipole and quadrupole polarizabilities published recently by Tunklev et al. (1997), i.e. $\alpha_{\mathrm{d}}=0.00921 a_{0}^{3}$ and $\alpha_{\mathrm{q}}=0.00111 a_{0}{ }^{5}$, and the ionization energy $E_{\mathrm{I}}=520175.9 \mathrm{~cm}^{-1}$ obtained by the same authors.

Table 1. Ritz formulae for the $n \mathrm{f}, n \mathrm{~g}$ series in C II and the $n \mathrm{~g}$ series in C III. The standard deviations between experimental term energies (Bashkin \& Stoner 1975) and those calculated using the Ritz formulae are also given

\begin{tabular}{llcccc}
\hline Ion & Series & $a$ & $b$ & $c$ & standard deviation \\
\hline C II & $n \mathrm{f}^{2} \mathrm{~F}^{\mathrm{o}}$ & 0.028958 & -0.221105 & 0.828474 & $0.50 \mathrm{~cm}^{-1}$ \\
C II & $n \mathrm{~g}^{2} \mathrm{G}$ & 0.006837 & -0.040060 & -0.242426 & $0.32 \mathrm{~cm}^{-1}$ \\
C III & $n \mathrm{~g}^{1,3} \mathrm{G}$ & 0.026138 & -0.672472 & 6.720485 & $0.41 \mathrm{~cm}^{-1}$ \\
\hline
\end{tabular}


The term energies calculated in our work are compared with available experimental values in Table 2. Examining this table, it is seen that the discrepancies between both sets of values are very small (the mean difference being $0.8 \mathrm{~cm}^{-1}$ ) if we except the $6 \mathrm{~h}^{2} \mathrm{H}^{\mathrm{o}}$ term belonging to $\mathrm{C}$ II. For this term, the difference between our value, calculated using the polarization formula, and the measured energy (Bashkin \& Stoner 1975) reaches $64.6 \mathrm{~cm}^{-1}$ which probably means that this particular term energy should be redetermined experimentally.

Tables 3, 4 and 5 contain the term energies adopted in the present study for the $n \mathrm{f}, n \mathrm{~g}, n \mathrm{~h}, n \mathrm{i}$ series in $\mathrm{C} \mathrm{II}$, for the $n \mathrm{~g}, n \mathrm{~h}, n \mathrm{i}, n \mathrm{k}$ series in C III and for the $n \mathrm{f}, n \mathrm{~g}, n \mathrm{~h}, n \mathrm{i}$, $n \mathrm{k}$ series in C IV respectively for $n$ up to 30 . Following the hereabove discussion, the mean uncertainty affecting our predicted values in these series should not exceed $1 \mathrm{~cm}^{-1}$.

Table 2. Comparison between the term energies calculated in this work and the available experimental data. All values are given in $\mathrm{cm}^{-1}$

\begin{tabular}{|c|c|c|c|c|}
\hline $\mathrm{n}$ & Гerm & Exp & This work & $\Delta$ \\
\hline & Af $2 \mathrm{~F}^{\circ}$ & & & \\
\hline & $2 \mathrm{~F}^{\mathrm{O}}$ & & & -0 . \\
\hline II & $6 \mathrm{f}^{2} \mathrm{~F}^{\mathrm{o}}$ & $4376.5^{a}$ & 184376. & 0.0 \\
\hline & $7 \mathrm{f}^{2} \mathrm{~F}^{\mathrm{o}}$ & & & \\
\hline & $5 \mathrm{~g}^{2} \mathrm{G}$ & & & 0. \\
\hline II & $6 \mathrm{~g}^{2} \mathrm{G}$ & & 1844 & .0 \\
\hline II & $7 \mathrm{~g}^{2} \mathrm{G}$ & & & 0.0 \\
\hline & $8 \mathrm{~g}^{2} \mathrm{G}$ & & & -1.3 \\
\hline II & $6 \mathrm{~h}^{2} \mathrm{H}^{\mathrm{o}}$ & & & \\
\hline II & $7 \mathrm{~h}^{2} \mathrm{H}^{\circ}$ & & & -1.2 \\
\hline & $5 \mathrm{~g}^{1} \mathrm{G}$ & & & -0.6 \\
\hline & $5 \mathrm{~g}^{3} \mathrm{G}$ & & & -0.6 \\
\hline & $6 \mathrm{~g}^{1} \mathrm{G}$ & & & -0.4 \\
\hline & $6 \mathrm{~g}^{3} \mathrm{G}$ & & & -0.3 \\
\hline & $7 \mathrm{~g}^{3} \mathrm{G}$ & & & -0.2 \\
\hline & $6 \mathrm{~h}^{3} \mathrm{H}^{\circ}$ & & & 0.5 \\
\hline II & $4 \mathrm{f}^{2} \mathrm{~F}^{\mathrm{o}}$ & 41 & & 0.2 \\
\hline IV & $5 \mathrm{f}^{2} \mathrm{~F}^{\mathrm{o}}$ & $449941.3^{b}$ & & 1.5 \\
\hline & $6 \mathrm{f}^{2} \mathrm{~F}^{\mathrm{o}}$ & & & \\
\hline & $7 \mathrm{f}^{2} \mathrm{~F}^{\mathrm{o}}$ & & & \\
\hline 1 & $8 \mathrm{f}^{2} \mathrm{~F}^{\mathrm{o}}$ & & & \\
\hline 18 & $5 \mathrm{~g}^{2} \mathrm{G}$ & & & 0. \\
\hline IV & $6 g^{2} \mathrm{G}$ & $471405.8^{b}$ & 4714 & 1. \\
\hline & $7 \mathrm{~g}^{2} \mathrm{G}$ & & & 1. \\
\hline & $8 \mathrm{~g}^{2} \mathrm{G}$ & & &. \\
\hline & $\mathrm{h}^{2} \mathrm{H}^{\mathrm{o}}$ & & & \\
\hline & $\mathrm{H}^{\circ}$ & & & \\
\hline & $8 \mathrm{~h}^{2} \mathrm{H}^{\mathrm{o}}$ & & & 1.4 \\
\hline & $9 \mathrm{~h}^{2} \mathrm{H}^{\circ}$ & 498 & 4985 & \\
\hline & & & & \\
\hline & & & & \\
\hline & & 40850 & $498500.2^{d}$ & \\
\hline
\end{tabular}

\footnotetext{
${ }^{a}$ Bashkin \& Stoner (1975).

$b$ Tunklev et al. (1997).

${ }^{c}$ Calculated using the Ritz formula.

${ }^{d}$ Calculated using the polarization formula.
}

Table 3. Adopted term energies (in $\mathrm{cm}^{-1}$ ) for the $n \mathrm{f}^{2} \mathrm{~F}^{\mathrm{o}}, n \mathrm{~g}$ ${ }^{2} \mathrm{G}, n \mathrm{~h}{ }^{2} \mathrm{H}^{\mathrm{o}}$ and $n \mathrm{i}{ }^{2} \mathrm{I}$ series of $\mathrm{C}$ II

\begin{tabular}{|c|c|c|c|c|}
\hline$n$ & $n \mathrm{f}^{2} \mathrm{~F}^{\circ}$ & $n \mathrm{~g}^{2} \mathrm{G}$ & $n \mathrm{~h}^{2} \mathrm{H}^{\circ}$ & $n \mathrm{i}^{2} \mathrm{I}$ \\
\hline & $168978.8^{a}$ & & & \\
\hline 5 & $178956.4^{a}$ & $179073.5^{a}$ & & \\
\hline 6 & $184376.5^{a}$ & $184449.7^{a}$ & $184529.9^{a}$ & \\
\hline 7 & $187642.0^{a}$ & $187691.8^{a}$ & $187701.0^{a}$ & $187705.1^{c}$ \\
\hline 8 & $189762.2^{b}$ & $189794.6^{a}$ & $189803.0^{c}$ & $189805.1^{c}$ \\
\hline 9 & $191214.0^{b}$ & $191238.2^{b}$ & $191243.3^{c}$ & $191244.8^{c}$ \\
\hline 10 & $192251.8^{b}$ & $192269.8^{b}$ & $192273.6^{c}$ & $192274.6^{c}$ \\
\hline 11 & $193019.2^{b}$ & $193032.9^{b}$ & $193035.8^{c}$ & $193036.6^{c}$ \\
\hline 12 & $193602.6^{b}$ & $193613.3^{b}$ & $193615.5^{c}$ & $193616.1^{c}$ \\
\hline 13 & $194056.4^{b}$ & $194064.9^{b}$ & $194066.6^{c}$ & $194067.1^{c}$ \\
\hline 14 & $194416.3^{b}$ & $194423.1^{b}$ & $194424.6^{c}$ & $194425.0^{c}$ \\
\hline 15 & $194706.6^{b}$ & $194712.2^{b}$ & $194713.3^{c}$ & $194713.7^{c}$ \\
\hline 16 & $194944.1^{b}$ & $194948.7^{b}$ & $194949.7^{c}$ & $194949.9^{c}$ \\
\hline 17 & $195140.9^{b}$ & $195144.7^{b}$ & $195145.5^{c}$ & $195145.7^{c}$ \\
\hline 18 & $195305.7^{b}$ & $195309.0^{b}$ & $195309.6^{c}$ & $195309.8^{c}$ \\
\hline 19 & $195445.2^{b}$ & $195448.0^{b}$ & $195448.5^{c}$ & $195448.7^{c}$ \\
\hline 20 & $195564.3^{b}$ & $195566.6^{b}$ & $195567.1^{c}$ & $195567.3^{c}$ \\
\hline 21 & $195666.7^{b}$ & $195668.8^{b}$ & $195669.2^{c}$ & $195669.3^{c}$ \\
\hline 22 & $195755.5^{b}$ & $195757.3^{b}$ & $195757.6^{c}$ & $195757.7^{c}$ \\
\hline 23 & $195832.9^{b}$ & $195834.5^{b}$ & $195834.8^{c}$ & $195834.9^{c}$ \\
\hline 24 & $195900.9^{b}$ & $195902.2^{b}$ & $195902.5^{c}$ & $195902.6^{c}$ \\
\hline 25 & $195960.8^{b}$ & $195962.0^{b}$ & $195962.3^{c}$ & $195962.4^{c}$ \\
\hline 26 & $196014.0^{b}$ & $196015.1^{b}$ & $196015.3^{c}$ & $196015.4^{c}$ \\
\hline 27 & $196061.3^{b}$ & $196062.3^{b}$ & $196062.5^{c}$ & $196062.6^{c}$ \\
\hline 28 & $196103.7^{b}$ & $196104.6^{b}$ & $196104.8^{c}$ & $196104.8^{c}$ \\
\hline 29 & $196141.8^{b}$ & $196142.5^{b}$ & $196142.7^{c}$ & $196142.8^{c}$ \\
\hline 30 & $196176.1^{b}$ & $196176.8^{b}$ & $196176.9^{c}$ & $196177.0^{c}$ \\
\hline
\end{tabular}

\section{Wavelengths and oscillator strengths}

The wavelengths in air, $\lambda$, and the oscillator strengths, $f$, for the $n \mathrm{~d}-n^{\prime} \mathrm{f}, n \mathrm{f}-n^{\prime} \mathrm{g}, n \mathrm{~g}-n^{\prime} \mathrm{h}, n \mathrm{~h}-n^{\prime} \mathrm{i}(n=3-6$, $\left.n^{\prime}=4-30\right)$ transitions in C II, the $n \mathrm{f}-n^{\prime} \mathrm{g}, n \mathrm{~g}-n^{\prime} \mathrm{h}$, $n \mathrm{~h}-n^{\prime} \mathrm{i}, n \mathrm{i}-n^{\prime} \mathrm{k}\left(n=4-7, n^{\prime}=5-30\right)$ transitions in C III and the $n \mathrm{~d}-n^{\prime} \mathrm{f}, n \mathrm{f}-n^{\prime} \mathrm{g}, n \mathrm{~g}-n^{\prime} \mathrm{h}, n \mathrm{~h}-n^{\prime} \mathrm{i}, n \mathrm{i}-n^{\prime} \mathrm{k}$ $\left(n=5-10, n^{\prime}=6-30\right)$ transitions in C IV are presented in Tables 6,7 and 8 respectively in the $400-1100 \mathrm{~nm}$ spectral region. The wavelengths were deduced from the $l \geq 3$ (C II), $l \geq 4$ (C III) and $l \geq 3$ (C IV) term energies reported in Tables $3-5$ and from the experimental energy levels published by Bashkin \& Stoner (1975) and Tunklev et al. (1997) for smaller values of the $l$-quantum number. From the estimated mean uncertainty affecting the term energies obtained in the present work (see Sect. 2.3 ), the predicted wavelengths in Tables $6-8$ should be accurate to within a few $0.01 \mathrm{~nm}$.

For the calculation of oscillator strengths, we used the relativistic Hartree-Fock (HFR) method with the help of the codes written by Cowan (1981). These computations were performed using the following sets of configurations: 
Table 4. Adopted term energies (in $\mathrm{cm}^{-1}$ ) for the $n \mathrm{~g}{ }^{1,3} \mathrm{G}, n \mathrm{~h}$ ${ }^{1,3} \mathrm{H}^{\circ}, n \mathrm{i}{ }^{1,3} \mathrm{I}$ and $n \mathrm{k}^{1,3} \mathrm{~K}^{\circ}$ series of C III

\begin{tabular}{|c|c|c|c|c|}
\hline$n$ & $n \mathrm{~g}^{1,3} \mathrm{G}$ & $n \mathrm{~h}^{1,3} \mathrm{H}^{\mathrm{o}}$ & $n \mathrm{i}^{1,3} \mathrm{I}$ & $n \mathrm{k}^{1,3} \mathrm{~K}^{\mathrm{o}}$ \\
\hline & $346579.3^{a}$ & & & \\
\hline 6 & $358692.2^{a}$ & $358776.3^{a}$ & & \\
\hline 7 & $365998.4^{a}$ & $366063.8^{c}$ & $366077.5^{c}$ & \\
\hline 8 & $370743.2^{b}$ & $370794.1^{c}$ & $370803.6^{c}$ & $370807.1^{c}$ \\
\hline 9 & $373997.4^{b}$ & $374037.0^{c}$ & $374043.8^{c}$ & $374046.4^{c}$ \\
\hline 10 & $376325.4^{b}$ & $376356.4^{c}$ & $376361.5^{c}$ & $376363.5^{c}$ \\
\hline 11 & $378047.8^{b}$ & $378072.5^{c}$ & $378076.3^{c}$ & $378077.8^{c}$ \\
\hline 12 & $379357.8^{b}$ & $379377.6^{c}$ & $379380.6^{c}$ & $379381.7^{c}$ \\
\hline 13 & $380377.1^{b}$ & $380393.2^{c}$ & $380395.5^{c}$ & $380396.5^{c}$ \\
\hline 14 & $381185.8^{b}$ & $381198.9^{c}$ & $381200.9^{c}$ & $381201.6^{c}$ \\
\hline 15 & $381838.1^{b}$ & $381849.0^{c}$ & $381850.6^{c}$ & $381851.2^{c}$ \\
\hline 16 & $382371.8^{b}$ & $382381.0^{c}$ & $382382.3^{c}$ & $382382.8^{c}$ \\
\hline 17 & $382814.1^{b}$ & $382821.8^{c}$ & $382822.9^{c}$ & $382823.3^{c}$ \\
\hline 18 & $383184.7^{b}$ & $383191.3^{c}$ & $383192.2^{c}$ & $383192.6^{c}$ \\
\hline 19 & $383498.3^{b}$ & $383503.9^{c}$ & $383504.7^{c}$ & $383505.0^{c}$ \\
\hline 20 & $383766.0^{b}$ & $383770.9^{c}$ & $383771.5^{c}$ & $383771.8^{c}$ \\
\hline 21 & $383996.3^{b}$ & $384000.6^{c}$ & $384001.1^{c}$ & $384001.4^{c}$ \\
\hline 22 & $384195.9^{b}$ & $384199.6^{c}$ & $384200.1^{c}$ & $384200.3^{c}$ \\
\hline 23 & $384370.1^{b}$ & $384373.3^{c}$ & $384373.8^{c}$ & $384373.9^{c}$ \\
\hline 24 & $384522.9^{b}$ & $384525.8^{c}$ & $384526.1^{c}$ & $384526.3^{c}$ \\
\hline 25 & $384657.7^{b}$ & $384660.3^{c}$ & $384660.6^{c}$ & $384660.7^{c}$ \\
\hline 26 & $384777.2^{b}$ & $384779.5^{c}$ & $384779.8^{c}$ & $384779.9^{c}$ \\
\hline 27 & $384883.8^{b}$ & $384885.8^{c}$ & $384886.1^{c}$ & $384866.2^{c}$ \\
\hline 28 & $384979.0^{b}$ & $384980.9^{c}$ & $384981.1^{c}$ & $384981.2^{c}$ \\
\hline 29 & $385064.6^{b}$ & $385066.3^{c}$ & $385066.5^{c}$ & $385066.6^{c}$ \\
\hline 30 & $385141.8^{b}$ & $385143.3^{c}$ & $385143.5^{c}$ & $385143.6^{c}$ \\
\hline
\end{tabular}

C II: $1 \mathrm{~s}^{2} 2 \mathrm{~s}^{2} n \mathrm{~d}(n=3-6)+1 \mathrm{~s}^{2} 2 \mathrm{~s}^{2} n \mathrm{~g}(n=5-30)+$ $1 \mathrm{~s}^{2} 2 \mathrm{~s}^{2} n i(n=7-30)+1 \mathrm{~s}^{2} 2 \mathrm{~s} 2 \mathrm{p}^{2}+1 \mathrm{~s}^{2} 2 \mathrm{~s} 2 \mathrm{p} 3 \mathrm{p}+1 \mathrm{~s}^{2} 2 \mathrm{~s} 2 \mathrm{p} 4 \mathrm{p}$ $+1 \mathrm{~s}^{2} 2 \mathrm{~s} 2 \mathrm{p} 4 \mathrm{f}$ (even parity) and $1 \mathrm{~s}^{2} 2 \mathrm{~s}^{2} n f(n=4-30)+$ $1 \mathrm{~s}^{2} 2 \mathrm{~s}^{2} n h(n=6-30)+1 \mathrm{~s}^{2} 2 \mathrm{~s} 2 \mathrm{p} 3 \mathrm{~d}+1 \mathrm{~s}^{2} 2 \mathrm{~s} 2 \mathrm{p} 4 \mathrm{~d}$ (odd parity);

C III: $1 \mathrm{~s}^{2} 2 \operatorname{sif}(n=4-7)+1 \mathrm{~s}^{2} 2 \mathrm{~s} n \mathrm{~h}(n=6-30)+1 \mathrm{~s}^{2} 2 \mathrm{~s} n \mathrm{k}$ $(n=8-30)+1 \mathrm{~s}^{2} 2 \mathrm{p} 3 \mathrm{~d}+1 \mathrm{~s}^{2} 2 \mathrm{p} 4 \mathrm{~d}$ (odd parity) and $1 \mathrm{~s}^{2} 2 \mathrm{~s} n \mathrm{~g}$ $(n=5-30)+1 \mathrm{~s}^{2} 2 \mathrm{~s} n \mathrm{i}(n=7-30)+1 \mathrm{~s}^{2} 2 \mathrm{p} 4 \mathrm{f}$ (even parity);

C IV: $1 \mathrm{~s}^{2} n \mathrm{~d}(n=3-10)+1 \mathrm{~s}^{2} n \mathrm{~g}(n=5-30)+1 \mathrm{~s}^{2} n \mathrm{i}$ $(n=7-30)$ (even parity) and $1 \mathrm{~s}^{2} n \mathrm{f}(n=4-30)+1 \mathrm{~s}^{2} n \mathrm{~h}$ $(n=6-30)+1 \mathrm{~s}^{2} n \mathrm{k}(n=8-30)$ (odd parity).

Scaling factors (0.90) were introduced for the Slater integrals while the ab initio spin-orbit parameters, calculated by the Blume-Watson method, were retained. Moreover, the average energies were adjusted using the experimental energy levels (Bashkin \& Stoner 1975; Tunklev et al. 1997) and predicted values reported in Tables $3-5$. Comparison with previously published oscillator strengths is possible only for transitions involving relatively small $n$-values. As an example, within the framework of the Opacity Project (OP), $f$-values for $n l-n^{\prime} l^{\prime}$ transitions $\left(n, n^{\prime} \leq 10, l, l^{\prime} \leq 4\right)$ were calculated by
Table 5. Adopted term energies $\left(\right.$ in $\mathrm{cm}^{-1}$ ) for the $n \mathrm{f}^{2} \mathrm{~F}^{\mathrm{o}}, n \mathrm{~g}$ ${ }^{2} \mathrm{G}, n \mathrm{~h}{ }^{2} \mathrm{H}^{\mathrm{o}}, n \mathrm{i}{ }^{2} \mathrm{I}$ and $n \mathrm{k}^{2} \mathrm{~K}^{\mathrm{o}}$ series of $\mathrm{C} \mathrm{IV}$

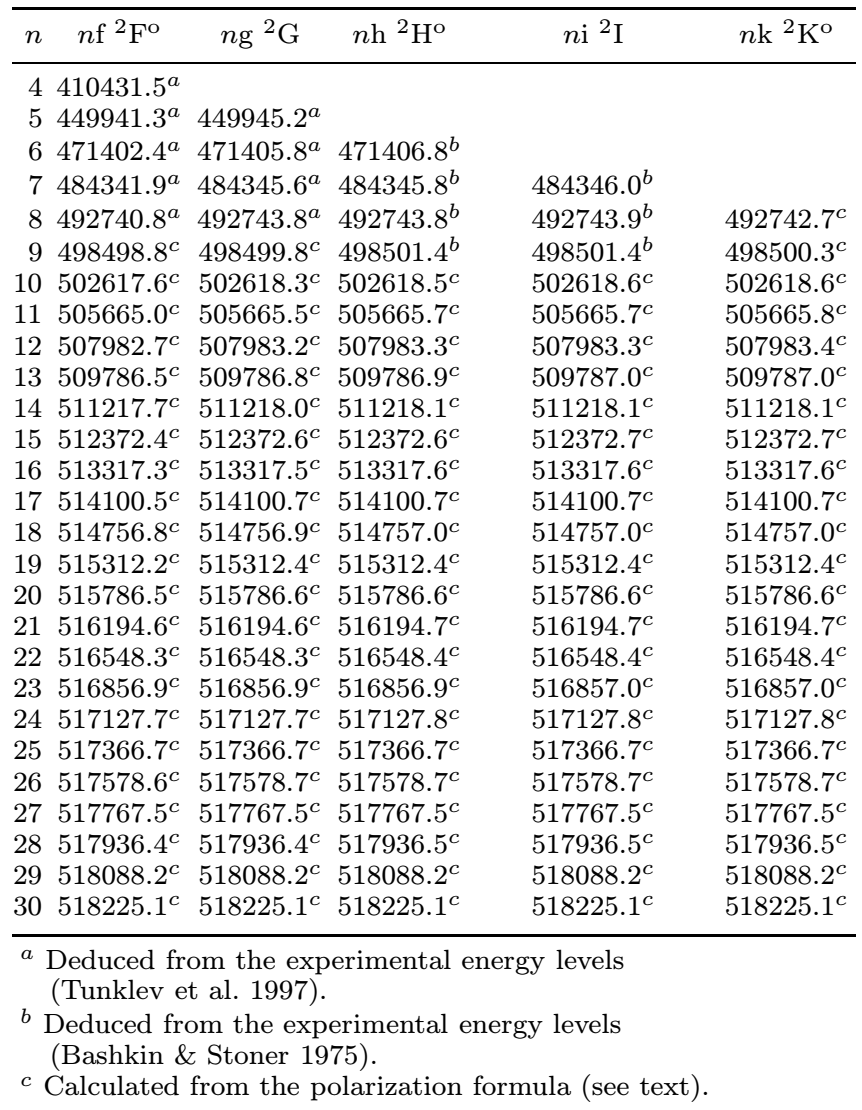

Fernley et al. (1997) and Tully et al. (1990) in the cases of C II and C III respectively and compiled in the TOPBASE atomic database (Cunto et al. 1993) at the CDS (http://cdsweb.u-strasbg.fr/OP.html). These OP oscillator strengths are compared in Fig. 1 with the results obtained in the present work. In general, the agreement between both sets of oscillator strengths is within a few percent if we except the $5 \mathrm{f}-7 \mathrm{~g}, 6 \mathrm{f}-8 \mathrm{~g}, 6 \mathrm{f}-9 \mathrm{~g}, 6 \mathrm{f}-10 \mathrm{~g}$ singlet and $5 \mathrm{f}-8 \mathrm{~g}$ triplet transitions of C III for which the discrepancies reach $25 \%$. An exception occurs also for the C III $5 \mathrm{f}^{1} \mathrm{~F}^{\circ}-8 \mathrm{~g}{ }^{1} \mathrm{G}$ line for which the $f$-value obtained in the present work is one order of magnitude smaller than the OP result. However, for this particular transition, our calculated line strength is affected by strong destructive interference effects (cancellation effects) indicating that the corresponding $f$-value may be expected to show a large error.

\section{Some astrophysical implications}

Some astrophysical implications of the new atomic data obtained in the present study are now discussed by means of a comparison with the observed spectrum of the planetary nebula NGC 7027 in the $654-1046 \mathrm{~nm}$ region. Possible 


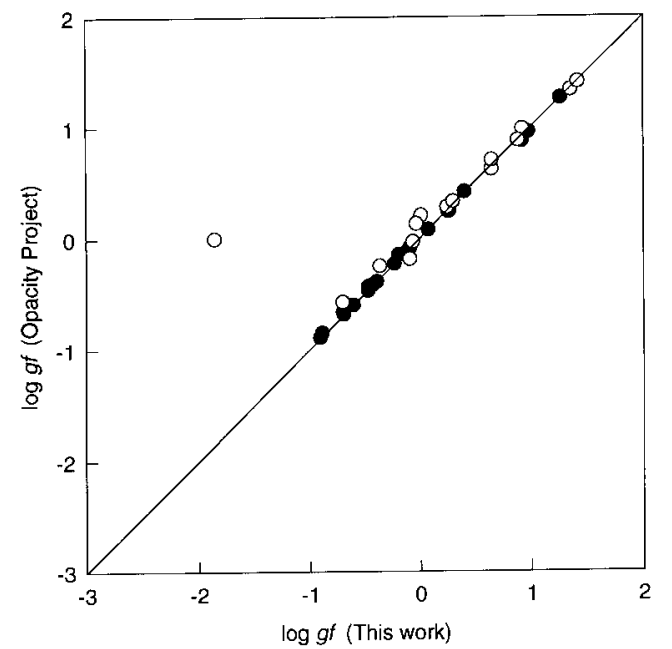

Fig. 1. Comparison between the oscillator strengths (log $g f)$ obtained in the present work and the Opacity Project results compiled in the TOPBASE atomic database (Cunto et al. $1993)$ for the $n \mathrm{~d}-n^{\prime} \mathrm{f}, n \mathrm{f}-n^{\prime} \mathrm{g}\left(n=3-5, n^{\prime}=4-10\right)$ transitions of C II $(\bullet)$ and the $n \mathrm{f}-n^{\prime} \mathrm{g}\left(n=4-7, n^{\prime}=5-10\right)$ transitions of C III (o)

observations of lines involving high- $n l$ Rydberg states in C II, C III and C IV are also predicted in the $\lambda<654 \mathrm{~nm}$ spectral region of this nebula.

\section{1. $C I I$}

The $4 \mathrm{~d}-5 \mathrm{f}$ and $4 \mathrm{f}-5 \mathrm{~g}$ lines, appearing at 922.89 and $990.39 \mathrm{~nm}$ respectively in the $654-1046 \mathrm{~nm}$ NGC 7027 spectrum (Baluteau et al. 1995) are strong enough to imply other $4 \mathrm{~d}-n \mathrm{f}$ and $4 \mathrm{f}-n \mathrm{~g}$ recombination lines should be detectable in the region $400-654 \mathrm{~nm}$. For these series, our predicted wavelengths are $615.14,512.22,462.03,432.98$, 414.35 and $401.58 \mathrm{~nm}$ for the $4 \mathrm{~d}-n \mathrm{f}(n=6-11)$ transitions and $646.20,534.24,480.27,449.12,429.23,415.61$ and $405.82 \mathrm{~nm}$ for the $4 \mathrm{f}-n \mathrm{~g}(n=6-12)$ lines. If we except the transitions $5 \mathrm{~d}-9 \mathrm{f}, 5 \mathrm{~g}-9 \mathrm{~h}, 5 \mathrm{~g}-11 \mathrm{~h}$ and $5 \mathrm{~g}-12 \mathrm{~h}$, blended or masked by other lines, all members of the series $5 \mathrm{~d}-n \mathrm{f}(n=8-11), 5 \mathrm{f}-n \mathrm{~g}(n=8-12)$ and $5 \mathrm{~g}-n \mathrm{~h}(n=$ $8-13$ ) are observed in the NGC 7027 spectrum published by Baluteau et al. (1995). However, in view of the relatively small calculated oscillator strengths corresponding to these transitions for larger $n$-values, only the first next members of these series could probably appear in the $\lambda<$ $654 \mathrm{~nm}$ spectral region. The transitions with $n=6$ lower terms, characterized by small $f$-values, are not observed in the NGC 7027 spectrum.

\subsection{III}

The large values of oscillator strengths for the $5 \mathrm{f}^{1} \mathrm{~F}^{\mathrm{o}}-$ $6 \mathrm{~g}^{1} \mathrm{G}(f=1.11)$ and $5 \mathrm{f}^{3} \mathrm{~F}^{\mathrm{o}}-6 \mathrm{~g}{ }^{3} \mathrm{G}(f=1.26)$ transitions observed in the Baluteau et al. (1995) NGC 7027 spectrum suggest higher members of these series might be detectable. The only $5 \mathrm{f}-n \mathrm{~g}$ transitions which might appear in the $400-654 \mathrm{~nm}$ spectral region are predicted at $583.32,456.84,530.51$ and $423.80 \mathrm{~nm}$ for $5 \mathrm{f}^{1}-7 \mathrm{~g}^{1}$, $5 \mathrm{f}^{1}-8 \mathrm{~g}^{1}, 5 \mathrm{f}^{3}-7 \mathrm{~g}^{3}$ and $5 \mathrm{f}^{3}-8 \mathrm{~g}^{3}$ respectively. The $5 \mathrm{~g}-6 \mathrm{~h}$ and $6 \mathrm{~g}-8 \mathrm{~h}$ lines appearing at 819.67 and $826.11 \mathrm{~nm}$ in the NGC 7027 spectrum are also characterized by large oscillator strengths ( $f=1.65$ and 0.267 respectively) which implies other lines from higher $n$ h levels could be observable. These lines should appear at the following predicted wavelengths: $513.09,412.86 \mathrm{~nm}$ for $5 \mathrm{~g}-n \mathrm{~h}(n=7$, 8), 651.51, 565.96, 515.85, 483.30, 460.68, 444.19, 431.72, $422.02,414.31,408.06$ and 402.92 for $6 \mathrm{~g}-n \mathrm{~h}(n=9-19)$. Some of the $7 \mathrm{~g}-n \mathrm{~h}, 7 \mathrm{~h}-n \mathrm{i}$ and $7 \mathrm{i}-n \mathrm{k}$ transitions are also observed for $n=10-14$ in the Baluteau et al. (1995) NGC 7027 spectrum. Consequently, wavelength predictions for higher members of these series up to $n=30$ are given in Table 7 .

\section{3. $C I V$}

In the $654-1046 \mathrm{~nm}$ region of the NGC 7027 spectrum, the highest members of the $8-n, 9-n$ and $10-n$ Rydberg series, detected by means of unblended lines, are situated at $773.57 \mathrm{~nm}(8-11), 674.69 \mathrm{~nm}(9-16)$ and $702.19 \mathrm{~nm}(10-23)$. Other lines in these series might be observable in the $400-654 \mathrm{~nm}$ region and their predicted wavelengths are reported in Table 8 up to $n=$ 26,30 and 30 for the $8-n, 9-n$ and $10-n$ series respectively. However, as already mentioned by Baluteau et al. (1995), all C IV lines involving quasi-hydrogenic levels with large principal quantum numbers should be systematically blended with homologous O IV and N IV lines.

\section{Conclusion}

Recent analyses of the spectrum of the planetary nebula NGC 7027 by Péquignot \& Baluteau (1994), Baluteau et al. (1995) and Péquignot (1996) have stimulated the present calculation of term energies, wavelengths and oscillator strengths for transitions involving Rydberg states of C II, C III and C IV for values of the principal quantum number up to $n=30$. Our results have been compared with the NGC 7027 spectrum observed in the $654-1046 \mathrm{~nm}$ region by Baluteau et al. (1995) and wavelength predictions to an accuracy of at least a few $0.01 \mathrm{~nm}$ have been obtained for lines which might be detectable in smaller wavelength regions. Several of our predicted lines coincide with many lines observed in the unpublished NGC 7027 spectrum between 400 and $654 \mathrm{~nm}$ (Péquignot 1997).

Acknowledgements. Financial support from the Belgian Fund for Scientific Research (F.N.R.S.) is acknowledged. It is also a pleasure to thank Dr. Péquignot (Meudon) for providing us with unpublished spectra of NGC 7027. 


\section{References}

Baluteau J.P., Zavagno A., Morisset C., Péquignot D., 1995, A\&A 303, 175

Bashkin S., Stoner J.O., 1975, Atomic Energy Levels and Grotian Diagrams, Vol. I. North Holland/American Elsevier

Connerade J.P., Farooq W.A., Ma H., Nawaz M., Shen N., 1992, J. Phys. B 25, 1405

Cowan R.D., 1981, The Theory of Atomic Structure and Spectra. University of California Press, Berkeley

Cunto W., Mendoza C., Ochsenbein F., Zeippen C.J., 1993, A\&A 275, L5

Drake G.W.F., 1994, Ad. At. Mol. Opt. Phys. 32, 93

Edlén B., 1964, Handbuch der Physik 27, Flugge S. (ed.). Springer, Berlin, p. 80

Fernley J.A., Hibbert A., Kingston A.E., Seaton M.J., 1997, J. Phys. B (to be published)

Hoang-Binh D., 1990, IAU Coll. 125 Radio recombination lines: 25 years of investigations, Gordon M.A., Sorochenko R.L. (eds). Kluwer, Dordrecht

Hoang-Binh D., 1993, A\&AS 97, 769

Hoang-Binh D., Brault P., Picart J., Tran-Minh N., Vallée O., 1987, A\&A 181, 134

Mende W., Kock M., 1996, J. Phys. B 29, 655

Moore C.E., 1970, Natl. Stand. Ref. Data Ser., NBS No. 34

Péquignot D., 1996, Phys. Scr. T65, 137

Péquignot D., 1997 (private communication)

Péquignot D., Baluteau J.P., 1994, A\&A 283, 593

The Opacity Project Team, 1995, The Opacity Project Volume 1. The Institute of Physics Publishing, Bristol and Philadelphia

Tully J.A., Seaton M.J., Berrington K.A., 1990, J. Phys. B 23, 3811

Tunklev M., Engstrom L., Jupén C., Kink I., 1997, Phys. Scr. 55,707

Verner D.A., Barthel P.D., Tytler D., 1994, A\&AS 108, 287 